\title{
Improving inpatient care with the introduction of a hip fracture pathway
}

\author{
Mark Chamberlain, Hannah Pugh
}

University College London Hospitals NHS Foundation Trust, UK

\begin{abstract}
\section{Problem}

University College London Hospitals (UCLH) NHS Foundation Trust provides an acute orthopaedic service for hip fractures, admitting approximately 120 patients annually. Despite an admission pathway being in place, UCLH performed badly in the 2013 national hip fracture database (NHFD) audit and was one of six hospitals not included in the annual report because insufficient information was submitted.[1] Of the recorded data, performance was poor against the agreed standards of care, which resulted in a low proportion of best practice tariff (BPT) payments and so a loss of income for the department.
\end{abstract}

A system of payment by results exists for the management of hip fractures in England and Wales. Poor performance against the national standards was noted, reflecting failure to deliver optimal care. Through the introduction of a multi-disciplinary patient pathway and clerking pro forma, the proportion of patients earning the best practice tariff uplift increased from $44.4 \%$ to $91.7 \%$. This demonstrates a significant improvement in patient care measured against the guidelines, also resulting in a substantial revenue increase for the department.

\section{Background}

Hip fractures are growing in incidence, with currently 70-75,000 per year in the UK. They present a massive financial burden on the healthcare system (estimated at £2 billion per annum) as well as a high associated mortality rate, with $10 \%$ of patients dying within one month.[2]

The NHFD is part of the Royal College of Physicians' falls and fragility fracture audit programme, and is a web-based audit of all 186 acute hospitals in England, Wales, and Northern Ireland, that assesses the management of patients aged 65 or older with hip fractures against nationally-agreed standards, including those from the British Orthopaedic Association and the British Geriatric Society's joint publication 'The care of patients with fragility fracture'[3], and the National Institute for Health and Care Excellence's (NICE) clinical guideline 'The management of hip fracture in adults'.[2]

The data submitted to the NHFD supports the payment by results BPT scheme, where a $£ 1,335$ uplift[4] can be awarded per patient for meeting nine criteria[5]: surgery within 36 hours of admission, shared care between orthopaedics and geriatrics, admission using an agreed assessment protocol, assessment by senior geriatrician within 72 hours, documentation of both pre- and post-operative abbreviated mental test (AMT) scores, geriatrician-led multidisciplinary rehabilitation, a falls assessment, and a bone health assessment.
Compliance with these standards has consistently been shown to be beneficial to patients:

- Friedman et al demonstrated improved outcomes by comanagement with geriatricians[6]

- Patel et al showed reduction in mortality with implementation of the NHFD standards[7]

- Thwaites et al reported an increase in patients receiving osteoporosis treatment with shared care[8]

- Gupta published a reduction in time to surgery with geriatrician input.[9]

Several papers have also reported a decreased length of stay following a multidisciplinary care protocol.[10-14]

\section{Baseline measurement}

A retrospective baseline audit was conducted on two months of hip fracture admissions from 2013. Patients were identified from NHFD records, and data gathered from their notes. Twenty hip fracture patients were identified in total, however two were excluded from the audit as they were aged below 65 . From the quarterly reports, BPT uplift was only achieved in eight of the 18 patients (44.4\%). Of the ten who did not reach minimum standards, four waited longer than 36 hours for surgery, seven waited longer than 72 hours for orthogeriatric review, and nine did not have both AMT scores recorded.

Other variables that are not included in the BPT criteria were also assessed in the audit: fascia iliaca ( $\mathrm{Fl}$ ) blocks were only administered in the emergency department (ED) in three of the 18 patients (16.7\%), only one had a pain score in ED documented, and only four had pain scores documented on admission to the ward. In addition, using a Nottingham hip fracture score (NHFS) $\geq 4$ (equating to $\mathrm{a}>5 \%$ predicted 30 -day mortality)[15], 15 of the 18 patients were identified as potentially benefiting from highdependency unit (HDU) level support post-operatively, although only eight (53.3\%) ended up being admitted. The mean length of stay in an acute ward was 18.9 days. 


\section{Design}

After researching methods used at neighbouring Trusts, the previously-used single-page checklist was redesigned into a clerking booklet. The resulting eight-page multidisciplinary pro forma contained sections for each BPT standard, focusing on which has been shown to improve performance[16]. For example, the AMT questions were incorporated allowing for easy application of the test, and so helping achieve the indicators identified as poorperforming in the initial audit.

NICE guidelines were also included in the booklet, such as pathways on the front page prompting ED clinicians to make a referral to anaesthetics if the patient was appropriate for a block, summaries of the NICE analgesia in hip fracture pathway, and sections adapted from the NICE clinical guidelines allowing for easy documentation of fragility fracture risk (CG146) and falls assessment (CG161). Checklists of essential pre-operative tasks were added, which have been demonstrated to improve the rate of completion of required jobs.[17] Additionally, the NHFS calculator was included, with prompts to book post-operative HDU beds at admission for those with a predicted 30 -day mortality $>5 \%$.

\section{Strategy}

A multi-disciplinary hip fracture care group was established, with representation from orthopaedics, geriatrics, rheumatology, anaesthetics, critical care, emergency medicine and therapies, with buy-in for the patient pathway confirmed. Dedicated twice-weekly consultant-led orthogeriatric ward rounds were implemented, and the pathway booklet included in the local induction for emergency department and orthopaedic juniors.

A small pilot of the booklet was undertaken over a one-week period following agreement from the hip fracture care group, where issues such as a lack of space to document FI block consent and nursing actions were identified. The booklet subsequently underwent several small changes to incorporate team members' suggestions.

\section{Results}

After full implementation of the pathway and admission booklet, the same two months in 2014 were re-audited prospectively. Thirty hip fractures were identified, however six were excluded from the BPT (four were under 65 years of age and two were treated nonoperatively). The booklet was used at admission in 27 of the 30 patients $(90 \%)$, however the ED section was only complete in 20 patients $(74.1 \%)$. Twenty two of the 24 eligible patients received the BPT uplift (91.7\%) with both not meeting the criteria of time to surgery as they required extensive pre-operative optimisation on ITU before being deemed fit for surgery. Importantly, all patients had both pre- and post-operative AMTS scores documented.

Of the 24 eligible patients, $\mathrm{FI}$ blocks were administered in ED in six patients (8.3\% increase). Pain scores were documented on admission in nine patients $(31.9 \%$ increase). Two of the six who received $\mathrm{FI}$ blocks had their pain recorded after 30 minutes (33.3\%) and 14 had their pain score recorded on admission to the ward (36.1\% increase). The NHFS was documented in 14 cases $(58.3 \%)$, which meant that 10 of the 15 patients $(66.7 \%)$ with a score $\geq 4$ were admitted to HDU post-operatively, an increase of $13.3 \%$.

The mean length of stay was 16.4 days in an acute bed, which failed to show statistical significance $(p=0.066)$ compared to the first audit cycle using the Mann-Whitney $\mathrm{U}$ test, as demonstrated in the papers referenced above. However, with a bed-day costing £303 at $\mathrm{UCLH}$, the 2.5 day reduction in length of stay would equate to a saving of over $£ 90,000$ if applied to all 120 admissions in a year.

\section{Lessons and limitations}

In designing the pathway, it became clear that given the many different teams involved in providing care to hip fracture patients, a multi-disciplinary team approach was essential with input from several services. As there was an institutional buy-in to improving performance within the national audit, we were readily met with enthusiasm from senior staff. Finding incentives for the junior staff proved harder however, but this was overcome by simplifying the admission process and promoting the time-saving element of the pathway.

\section{Conclusion}

The resulting $47.3 \%$ increase in patients receiving the BPT uplift as a result of the procedural changes reflects a significant improvement in the delivery of optimal care. The introduction of the pathway and admission booklet resulted in a higher compliance with national standards. Based on an estimate of 120 cases a year eligible for the BPT, this also represents an additional income of $£ 75,700$ per annum for the department.

\section{References}

1. Clinical Effectiveness and Evaluation Unit, National Hip Fracture Database National report 2013, London: Royal College of Physicians.

2. NICE, 2011. CG124 - Hip fracture: The management of hip fracture in adults. Available at: https://www.nice.org.uk/guidance/cg124/ [Accessed 7 Jan 2015].

3. British Orthopaedic Association, 2007. The Care of Patients with Fragility Fracture.

4. Monitor, National tariff payment system 2014/15. Available at: https://www.gov.uk/government/publications/nationaltariff-payment-system-2014-to-2015 [Accessed 7 Jan 2015].

5. NICE, 2012. CMG46 - Management of hip fracture in adults. Available at:

https://www.nice.org.uk/guidance/cmg46/chapter/33-qualitymeasures [Accessed 7 Jan 2015].

6. Friedman SM, M. D. B. K. K. S. Impact of a comanaged Geriatric Fracture Center on short-term hip fracture outcomes. Arch Intern Med 2009;169(18):1712-7.

7. Patel NK, S. K. J. S. L. C. M. F. Implementing the National Hip Fracture Database: An audit of care. Injury 
2013;44(12):1934-9.

8. Thwaites JH, M. F. G. N. F. C. R. A. S. R. Shared care between geriatricians and orthopaedic surgeons as a model of care for older patients with hip fractures. N Z Med J 2005;118(1214):U1438.

9. Gupta A. The effectiveness of geriatrician-led comprehensive hip fracture collaborative care in a new acute hip unit based in a general hospital setting in the UK. J R Coll Physicians Edinb 2014;44(1):20-6.

10. González-Montalvo JI, A. T. M. J. G.-G. E. G. P. M.-V. A. The orthogeriatric unit for acute patients: a new model of care that improves efficiency in the management of patients with hip fracture. Hip Int 2010;20(2):229-35.

11. Kumar G. Protocol-guided hip fracture management reduces length of hospital stay. $\mathrm{Br} \mathrm{J}$ Hosp Med (Lond) 2012;73(11):645-8.

12. Flikweert ER, I. G. K. B. S. M. W. K. The development of a comprehensive multidisciplinary care pathway for patients with a hip fracture: design and results of a clinical trial. BMC Musculoskelet Disord 2014;(15):188.

13. Lau TW, F. C. L. F. The effectiveness of a geriatric hip fracture clinical pathway in reducing hospital and rehabilitation length of stay and improving short-term mortality rates. Geriatr Orthop Surg Rehabil 2013;4(1):3-9.

14. Suhm N, K. R. S. P. W. Q. K. R. R. D. J. M. P. M. Orthogeriatric care pathway: a prospective survey of impact on length of stay, mortality and institutionalisation. Arch Orthop Trauma Surg 2014;134(9):1261-9.

15. Moppett IK, P. M. G. R. B. T. W. S. M. C. Nottingham Hip Fracture Score: longitudinal and multi-assessment. $\mathrm{Br} \mathrm{J}$ Anaesth 2012;109(4):546-50.

16. Khan SK, W. A. B. S. T. A. P. A. The Best Practice Tariff helps improve management of neck of femur fractures: a completed audit loop. Br J Hosp Med (Lond) 2013;74(11)644-7.

17. Agha R, E. E. F. A. Improving the preoperative care of patients with femoral neck fractures through the development and implementation of a checklist. BMJ Qual Improv Report 2014;(3).

\section{Declaration of interests}

Nothing to declare.

\section{Acknowledgements}

UCLH Hip Fracture Care Group 